\title{
Objective measurement of upper limb activity and mobility during everyday behavior using ambulatory accelerometry: The Upper Limb Activity Monitor
}

\author{
FABIËNNE C. SCHASFOORT and J. B. J. (HANS) BUSSMANN \\ Erasmus MC, Rotterdam, The Netherlands \\ WIM L. J. MARTENS \\ TEMEC Instruments, Kerkrade, The Netherlands \\ and \\ HENK J. STAM \\ Erasmus MC, Rotterdam, The Netherlands
}

\begin{abstract}
Ambulatory accelerometry is a technique that allows objective measurement of aspects of everyday human behavior. The aim of our research has been to develop, validate, and apply this technique, which recently resulted in an upper limb activity monitor (ULAM). The ULAM consists of body-mounted acceleration sensors connected to a waist-worn data recorder and allows valid and objective assessment of activity of both upper limbs during performance of also automatically detected mobility-related activities: lying, sitting, standing, walking, cycling, and general movement. The ULAM can be used to determine (limitations of) upper limb activity and mobility in freely moving subjects with upper limb disorders. This article provides a detailed description of its characteristics, summarizes the results of a feasibility study and four application studies in subjects having upper limb complex regional pain syndrome, discusses the most important practical, technical, and methodological issues that were encountered, and describes current and future research projects related to measuring (limitations of) upper limb activity.
\end{abstract}

In experimental research and clinical practice, numerous techniques are used to measure different aspects of human behavior. Nonambulatory techniques have several disadvantages, the main ones being that measurements are usually short term and susceptible to "subjective" influences. From the perspective of rehabilitation medicine, the consequences of disease or disorder for subjects' everyday lives are very important. Being able to objectively measure (limitations of) activity during everyday behavior and activity generally has added value for diagnosis, therapy choice, evaluation of recovery, and determination of treatment effects. Due to developments in data recording and sensor technology, monitoring systems that measure aspects of human functioning and behavior have become available. Ambulatory accelerometry, for example, allows long-term objective measurement of what free-living subjects do during everyday life. Important characteristics of ambulatory accelerometry are the following: Subjects are measured in their home environment; natural behavior is,

Correspondence concerning this article should be addressed to F. C. Schasfoort, Department of Rehabilitation Medicine, Erasmus MC, P.O. Box 1738, 3000 DR Rotterdam, The Netherlands (e-mail: f.schasfoort@ erasmusmc.nl). therefore, measured; measurements are long term, with continuous data collection; data are unobtrusive and objective; and what subjects actually do, and not what they are capable of, is measured.

The aim of our research was to develop, validate, and apply ambulatory accelerometry for measuring during the everyday life of healthy and disabled subjects. This has resulted in two instruments: the Activity Monitor (AM) and the Upper Limb Activity Monitor (ULAM). Both instruments consist of body-mounted piezoresistive acceleration sensors connected to a portable data recorder. The AM allows valid automatic and objective detection of the mobility-related activities of lying, sitting, standing, walking, cycling, and general (noncyclic) movement and can be used to determine (limitations of) mobility (Bussmann, 1998; Bussmann et al., 2001; Bussmann, Veltink, Koelma, van Lummel, \& Stam, 1995). Although extensively validated for general mobility (H. B. Bussmann, Reuvekamp, Veltink, Martens, \& Stam, 1998; Bussmann, Tulen, van Herel, \& Stam, 1998; Bussmann, van de Laar, Neeleman, \& Stam, 1998; van den Berg-Emons, Bussmann, Balk, \& Stam, 2000), the AM was not adequate for patient groups having upper limb disorders. Limitations in the activity of these patient groups are directly related to upper limb usage during everyday life and behavior and 
are not primarily expressed in mobility-related activities. To enable detection of limited arm-hand usage of subjects with upper limb disorders, the possibilities of the AM were extended, resulting in the ULAM (Schasfoort, Bussmann, \& Stam, 2002). The ULAM allows objective measurement of the activity of both upper limbs during performance of mobility-related activities.

The aims of this article are (1) to provide a detailed description of ULAM characteristics, (2) to summarize the results of a ULAM feasibility study and four application studies in subjects having upper limb complex regional pain syndrome (CRPS), (3) to discuss practical, technical, and methodological issues that arise when the (UL)AM is used, and (4) to give an overview of current and future research projects related to measuring (limitations of) upper limb activity.

\section{Characteristics of ULAM}

The basic ULAM configuration consists of five bodymounted uni- and biaxial piezoresistive acceleration sensors $($ ADXL202, $1.5 \times 1.5 \times 1.0 \mathrm{~cm}$; Analog Devices, Breda, the Netherlands; adapted by TEMEC Instruments, Kerkrade, the Netherlands) that are connected to a portable data recorder (see Figure 1). The raw acceleration signals yielded by the piezoresistive accelerometers are a combination of a component of the gravitational acceleration $\left(9.81 \mathrm{msec}^{-2}\right)$ and a component of the inertial acceleration (Bussmann et al., 2001). The sensors are fixed on Rolian Kushionflex (Smith \& Nephew, Hoofddorp, the Netherlands) or silicone-based stickers (Schwa-Medico, Ehringshausen, Germany) by double-sided tape; both ma-

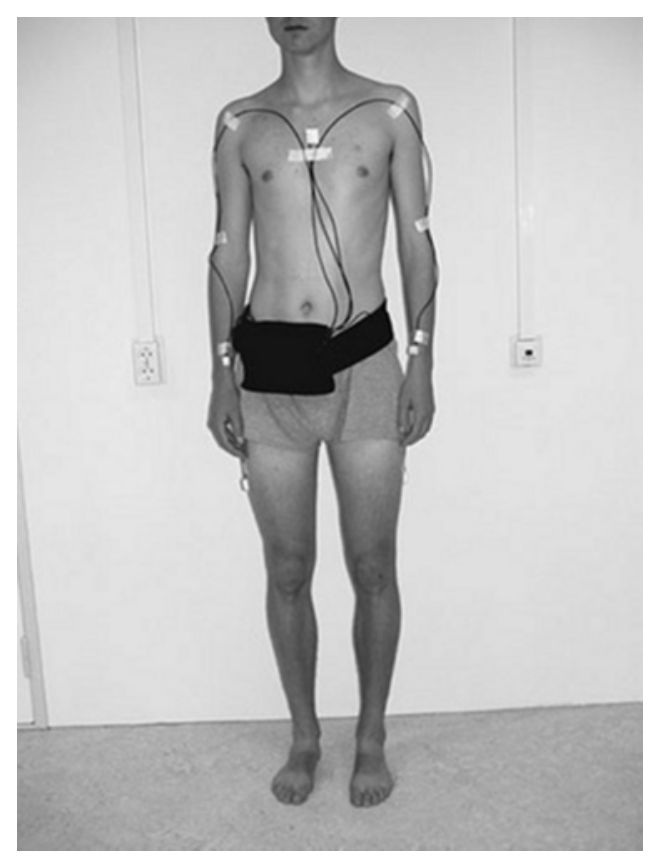

Figure 1. A subject wearing the Upper Limb Activity Monitor, with accelerometers on the forearms, trunk, and thigh(s) and the data recorder in a waist-worn machine-washable bag. terials can be fixed directly on the skin. With the subject standing in the anatomical position, two uniaxial sensors are attached to the forearms (i.e., one on each upper limb), just proximal from the wrist joint, with the sensitive axes in the sagittal direction; a biaxial sensor is attached on the lower part of the sternum, with sensitive axes in the sagittal and longitudinal direction, and one (or two) uniaxial sensor(s) is (are) attached at the lateral side of the thigh(s), with their sensitive axes in the sagittal direction. Signals from the five (or six) acceleration sensors are continuously measured and digitally stored (24-72 h) and, after the measurements have been made, downloaded onto a PC for analysis.

The automated kinematic analysis after a measurement has been made takes place by proprietary signalprocessing and inferencing language (SPIL), yielding C-code (Jain, Martens, Mutz, Weiss, \& Stephan, 1996). The first part of the analysis is feature extraction, which means that three new feature signals with specific characteristics are derived from each raw acceleration signal: the low-pass (LP)/angular feature, the motility feature, and the frequency feature. The second part of the analysis for the trunk and thigh signals consists of a 1-sec classification of mobility-related activities on the basis of these feature signals. These parts of the analysis, including (optional) postprocessing procedures, have been extensively described previously (Bussmann et al., 2001).

The detection of upper limb (in)activity takes place after the detection of mobility-related activities. The ULAM data analysis consists of several SPIL routines (Schasfoort et al., 2002). The first step is the actual detection of upper limb (in)activity, which is done for both upper limbs separately and is based on the motility feature of the forearm signals. The value of the motility feature signal depends on the variability of the raw signal around the mean. This variability can be regarded as a measure for upper limb activity; the more upper limb activity, the more the signal is varied, and the higher the motility value. The second analysis step generates a new signal for each forearm sensor that indicates per second whether there is upper limb activity or not, with different preset motility thresholds for different mobility-related activities (see also Figure 2). Descriptive statistics of (UL)AM outcome measures are automatically created for each measurement in a report. For both upper limb sensors, for example, the report displays the number of seconds that the individual upper limbs were active or inactive, as well as the mean (scaled) motility values for each of the mobility-related activities separately. It is important to note that the report may comprise the whole measurement period or one or more parts of it (e.g., nighttime vs. morning, afternoon, and/or evening).

\section{ULAM Feasibility and Application Studies}

In a feasibility study (Schasfoort et al., 2002), we assessed ULAM's ability to discriminate between (different forms of) upper limb usage and nonusage in 4 healthy subjects and 4 subjects with an upper limb disorder. The subjects performed an activity protocol representing sev- 


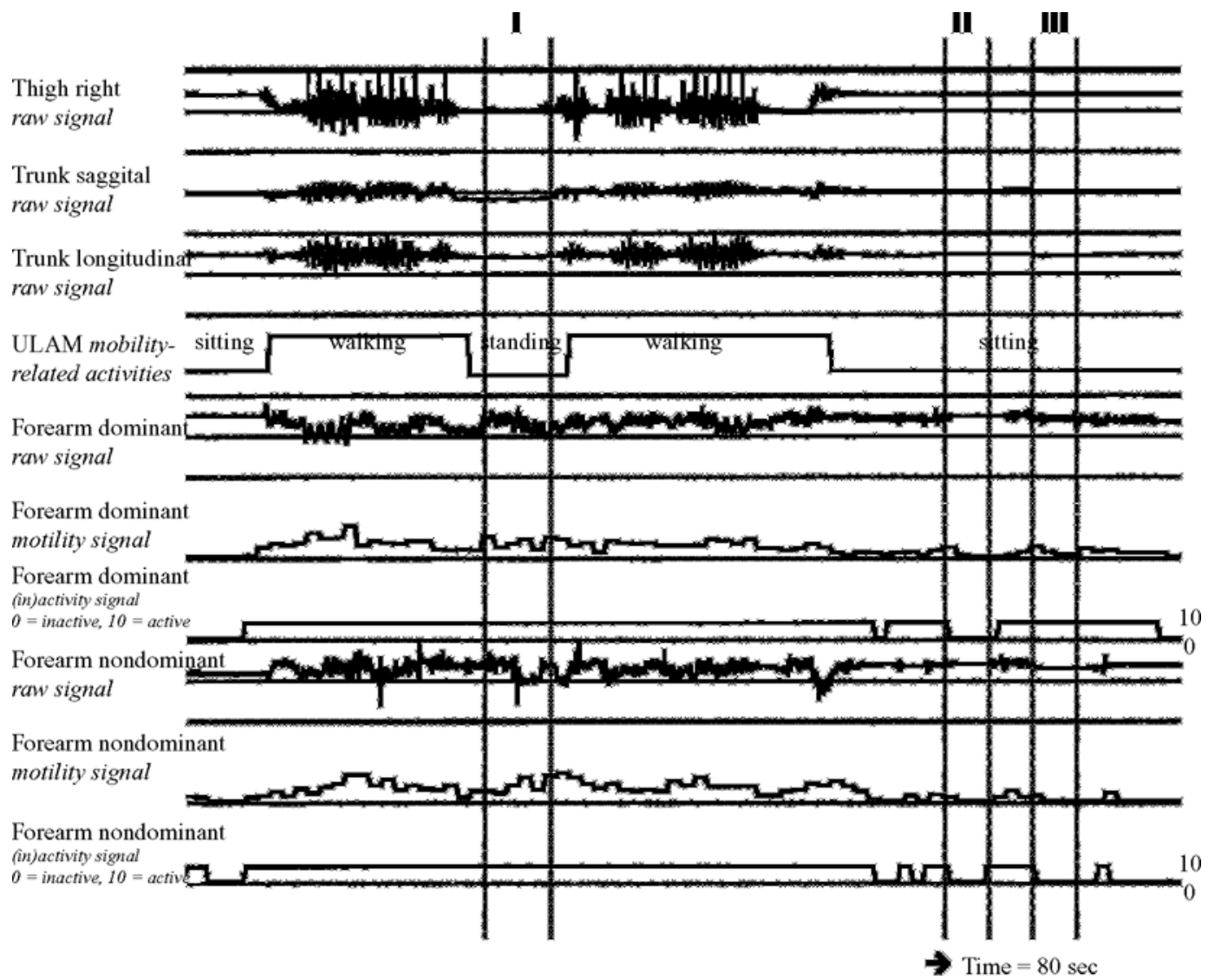

Figure 2. An example of 80 sec of several ULAM (feature) signals and the automatically detected output of mobility-related activities and upper limb (inactivity of the dominant and nondominant forearms of a healthy subject. The main ULAM outcome measures that were used for statistical analyses in the application studies are composed of the signals of the mobility-related activities and one or more of the forearms signals (1-sec resolution). Interval I (vertical dotted lines) shows that the intensity of activity of both upper limbs is relatively high (motility signals) and that both upper limbs are active $100 \%$ of the time while the subject is standing. The proportion of upper limb activity of one side, relative to the other, is, therefore, equal during the seconds in Interval I. Interval II shows that while the subject is sitting, both upper limbs have a lower intensity of activity, in comparison with Interval I, and that both upper limbs are inactive nearly $100 \%$ of the time. The proportion of upper limb activity of one side, relative to the other, is, therefore, equal in Interval II. Interval III shows that while the subject is sitting, the dominant upper limb has a higher intensity of upper limb activity and is active a higher percentage of the time than is the nondominant upper limb. The normalized dominant:nondominant side proportion for the percentage of upper limb activity will, therefore, be higher than 1. From "Impact of Upper Limb Complex Regional Pain Syndrome Type I on Everyday Life Measured With a Novel Upper Limb-Activity Monitor,” by F. C. Schasfoort, J. B. J. Bussmann, A. M. A. J. Zandbergen, and H. J. Stam, 2003, Pain, 101, p. 82. Copyright 2003 by Elsevier. Adapted with permision.

eral forms of real-life upper limb (non)usage as described in a framework (see Figure 3). The framework was based on our definition of upper limb usage as active movement of (parts of) the upper limb(s) in relation to proximal parts, holding objects, and/or leaning. Agreement scores between the ULAM and the video recordings (reference method) were calculated. It appeared that there were no systematic differences in the agreement percentages between healthy and disabled subjects but that the different forms of upper limb usage and nonusage were not equally well detected. Primary functional usage and plain nonusage without movement were well detected in $82.2 \%$ and $86.7 \%$, respectively, of their total duration. Secondary functional usage was always well detected (100\%).
Detection of upper limb usage without movement during leaning and holding and of involuntary/passive nonusage with movement was less than optimum, with agreement percentages of $37 \%$ and $23.9 \%$, respectively. At first sight, these findings may seem somewhat disappointing. However, for the activity protocol, mainly critical activities (i.e., activities with those forms of upper limb usage that were anticipated to be less well detected) were selected, which inevitably made agreement lower. The percentage of agreement for primary functional usage appeared to be "only" $82.2 \%$, because of wrong detection during manipulative (fine) movements and specific critical handling (gross) movements (e.g., turning the pages of a book, eating soup, or pouring water). When the forms of upper limb 


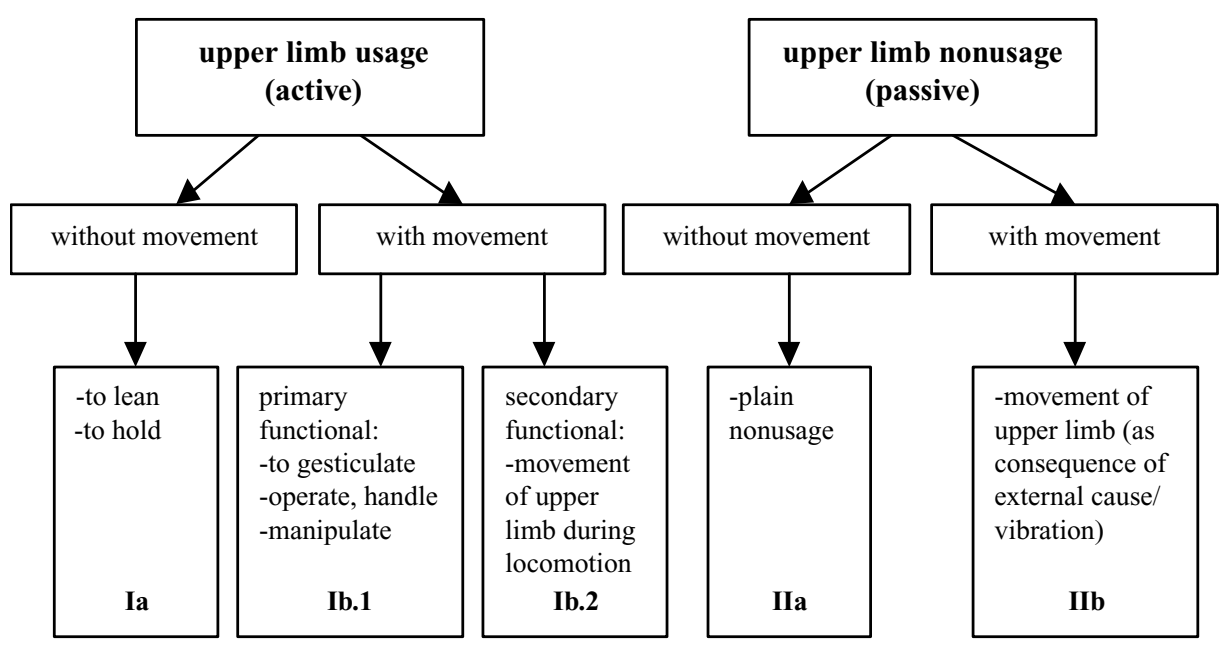

Figure 3. Overview of the different classes of real-life upper limb usage and nonusage based on the following definition of upper limb usage: active movement of (parts of) the upper limb(s) in relation to proximal parts, holding objects, and/or leaning. From "Ambulatory Measurement of Upper Limb Usage and Mobility-Related Activities During Normal Daily Life With an Upper Limb-Activity Monitor: A Feasibility Study," by F. C. Schasfoort, J. B. Bussmann, and H. J. Stam, 2002, Medical Biological Engineering and Computing, 40, p. 174. Copyright 2002 by Springer-Verlag. Adapted with permission.

(in)activity were combined with specific mobility-related activities, however, it appeared that the ULAM outcome measures that were of special interest from a rehabilitation point of view were satisfactorily detected (overall agreement, $83.9 \%$ ). It was, therefore, considered feasible to use ULAM in future studies in patients with an upper limb disorder.

The patient group that participated in the four ULAM application studies were subjects having unilateral upper limb CRPS Type I (also known as reflex sympathetic dystrophy). CRPS is a poorly understood and variously defined symptom complex that, when it occurs, usually follows surgery or trauma. CRPS is generally expressed in the extremities, and the upper limb is involved in about $50 \%$ of the cases. Sensory, autonomic, trophic, and motor impairments of body tissue and function may be found in CRPS, as well as activity limitations during everyday behavior and participation problems. Limitations of mobility-related activities and upper limb activity had not been measured objectively in this patient group before development of ULAM (Schasfoort, Bussmann, \& Stam, 2000). The main ULAM outcome measures that were used for statistical analyses in the application studies were the intensity of upper limb activity, expressed as the mean motility value (in $\mathrm{msec}^{-2}$ ); the percentage of upper limb activity, expressed as a percentage of the time the upper limb activity exceeded a motility threshold (activity duration in percentage); and the proportion of upper limb activity of one side, relative to the other, expressed as a ratio for the percentage of upper limb activity, each during the time a subject was sitting and standing (see also Figure 2). The percentages of dynamic mobility-related activities performed (i.e., walking, cycling, and general noncyclic movement), expressed as percentages of the 24-h measurement period, were used as outcome measures for general mobility.

In the first clinical application study (Schasfoort, Bussmann, Zandbergen, \& Stam, 2003), the long-term impact of CRPS on upper limb usage during everyday life, as measured with ULAM, was determined. In 10 female chronic patients (on average, 3.7 years after onset) and 10 matched healthy control subjects, 24-h ULAM activity patterns were measured. The main ULAM outcome measures described above were compared between the patients and the controls, and it appeared that there were no differences between the patients and the controls regarding general mobility but that there were marked differences regarding upper limb activity, especially for upper limb activity during sitting. Especially, the 5 patients with CRPS in their dominant side clearly showed less activity of their involved limb, in comparison with their controls, indicated by significant differences for the mean intensity $(p=.014)$, percentage $(p=.004)$, and proportion $(p=.032)$ of upper limb activity during sitting. These chronic CRPS patients still had objectively measurable limitations in upper limb usage during everyday activities and behavior.

The second application study (Schasfoort, Bussmann, \& Stam, 2004) focused on the important, for rehabilitation medicine, so-called impairment-activity-limitations relationship. Studying this relationship is important for addressing such questions as, which impairment particularly affects everyday activity? Thirty chronic CRPS subjects volunteered to participate, and several instruments were used to measure several impairments: temperature differences between the two hands (infrared thermometer), pain resulting from effort (VAS), pain during previous days (McGill Pain Questionnaire), differences in the maximum 
active range of motion (AROM) of the wrist and fingers between the two hands (goniometer), volume differences between the two hands (volumeter fluid overflow), and differences in grip strength between the two upper limbs (hand-held dynamometer). It was found that the involved upper limb was clearly less active than the noninvolved side, as indicated by significantly lower intensity $(p=$ .001 and $p=.001)$ and percentage $(p=.008$ and $p=$ $.002)$ of upper limb activity during sitting and standing, respectively. These upper limb activity limitations were again more prominent when the dominant side was involved. As for the impairment-activity-limitations relationship, a linear regression analysis showed that the variability in upper limb outcome measures explained by the six impairments and by age ranged from $24 \%$ to $52 \%$. Impaired active range of motion, grip strength, and, to a lesser extent, pain resulting from effort were the most important impairments explaining variance in everyday activity. The more impairments a subject had-and especially, motor impairments - the more objectively measured activity limitations were present.

Because ULAM clearly differs from commonly used techniques in research and clinical application with respect to several methodological and practical criteria, its place in the field of instruments measuring functioning and health was determined in the third application study (Schasfoort, Bussmann, \& Stam, 2005). In a cross-sectional comparison study, 30 patients with chronic CRPS were measured with ULAM (24 h) and subsequently completed four questionnaires, including two generic questionnaires (SIP68 and RAND36) and two body-part-specific questionnaires (DASH and RASQ). Spearman rank correlations were calculated between several outcome measures, and it appeared that $83 \%$ of the interquestionnaire correlations were significant, whereas $46 \%$ of the correlations between ULAM and the questionnaires were significant. The relationships between ULAM and questionnaire outcome measures were generally nonsignificant or weak. However, the more characteristics ULAM had in common with other instruments, the stronger and more often significant were the relationships that were found. ULAM measures similar aspects of functioning only to a certain extent, and it measures, at least partly, different areas of functioning and health.

In the fourth application study (Schasfoort, Bussmann, Krijnen, \& Stam, 2006), we explored upper limb activity over time, as objectively measured with ULAM, in 4 subjects with acute CRPS in one of the upper limbs. Four subjects were measured four times during their treatment. The upper limb activity time course, as measured with ULAM, was compared with the time course of other outcome measures for activity (limitations) and impairments. The main ULAM outcome measures were used (with the exception of the proportion of upper limb activity), as were three questionnaires for activity (limitations) (RASQ, DASH, and RAND36) and the six impairment outcome measures from the second application study. The results showed that objectively measured upper limb activity often improved; improvements of $>5 \%$ were found for the majority $(63 \%)$ of ULAM outcome measures at final assessment. Thus, we were able to detect clear changes in upper limb activity over time, as measured with ULAM, and the relationships between the time courses of the ULAM outcome measures and the other outcome measures for activity limitations and impairments were explainable.

\section{Advantages and Surplus Value of ULAM}

The objectively measured ULAM findings from the application studies were not always in concordance with subjective patient and clinician findings or opinions. The surplus value of a device such as ULAM, therefore, is that it enables one to study such discrepancies between objective and subjective findings, which are more regularly present than one often thinks. Second, there are no ceiling or floor effects that may hamper data interpretation, because ULAM measures what subjects actually do, whereas questionnaires, for example, measure functioning as perceived and recalled by subjects.

The ULAM configuration and its outcome measures also enable differentiation between the impact of dominant side involvement and that of nondominant side involvement on daily behavior in patient groups with upper limb disorders, a valuable topic that has not been investigated objectively in the home environment. In addition, ULAM can be used to compare activity limitations across patient groups with different upper limb disorders, because it is not a disease-specific instrument.

ULAM findings may also have added value for therapy choice, because the device allows objective determination of which treatment components have the highest potential to improve or maintain activity levels in patients with upper limb disorders. ULAM can also be used in research as one of the instruments in a set of instruments that differ with respect to measuring (aspects of) a subject's capacity or performance. This type of research would then contribute to the performance-versus-capacity discussion, which is a hot topic in behavioral research and, especially, in rehabilitation medicine. Finally, ULAM has the potential to validly assess upper limb activity over time in acute upper limb CRPS and can, therefore, be useful and valuable in longitudinal studies on the evaluation of (natural) recovery or to determine treatment effects in patient groups with upper limb disorders.

\section{Practical, Technical, and Methodological Considerations}

In our opinion, the application studies in CRPS demonstrate that ULAM offers an alternative, relevant, and important insight into the impact a disorder may have on a subject's functioning and behavior. In general, subjects do not consider wearing ULAM to be uncomfortable. The technique of ambulatory accelerometry has many advantages, but of course, there also are some practical, technical, and methodological issues that need consideration. To make use of ULAM easier and more comfortable, the recorder size should be decreased, and making the sen- 
sors wireless would be a great advantage. Some subjects disliked the "tourist look" of the waist bag, were unhappy with the fact that they can wash themselves only with a washcloth and sink during the measurement period, or had some objections to shaving off hair. For the future, the recorder and sensors should be more water resistant and, preferably, waterproof, which would allow extension of the measurement period. As for the sensor fixatives, there were hardly any problems with the current materials; most subjects considered them skin friendly. Only in cases of excessive sweating or an allergy to fixative materials or latex in the wires did we have to improvise. It also has to be noted that for subjects with pain in the upper limbs(s), concessions sometimes have to be made with respect to proximal-distal sensor attachment. As for the signal analysis, the SPIL-routines-based software package is currently being transformed into a more universally applicable and user friendly software package based on a multi-time-base environment with XML databases. Just like all medical and behavioral research, research with ULAM is subject to medical ethics committee guidelines. The ULAM output is no more and no less than a specific set of outcome measures related to mobility-related activities and upper limb activity; such activities as washing the dishes and waiting in line at the supermarket will be both standing and (greater or smaller) upper limb activities.

Because ULAM is, in fact, an extension of AM, several technical limitations that have already been discussed with respect to AM (Bussmann et al., 2001) also apply to ULAM. The acceleration sensors that we currently use are nondrifting and robust. To minimize interference with normal everyday activity patterns, ULAM is fitted in the subjects' home environment; correct alignment of the sensors is important. In view of the technique used, it was logical that the holding of objects and leaning were the most difficult forms of upper limb usage to detect. That is why we made the assumption that in normal upper limb usage, leaning and holding, as well as primary functional manipulation, are usually preceded and followed by active upper limb movements that bring the upper limb into the right position to lean, hold, or manipulate. So, even though ULAM did not allow valid measurement of every aspect of upper limb usage, we thought that if the ULAM outcome measures were defined in such a way that upper limb movement or activity is measured, it would be allowed to make a statement on the degree of limitations of subjects with an upper limb disorder. It has to be noted, however, that making this assumption does not mean that we will not work on optimizing detection of the different forms of upper limb usage or mobility-related activities. Optimizing detection is especially important when ULAM is to be validated and applied in other patient categories with clinical and "behavioral" problems different from those of the CRPS population. Developing and validating a device such as ULAM clearly is an ongoing, innovative, and, for that reason, complex and time-consuming process. However, the concept of using ambulatory monitoring techniques to determine (limitations of) activity and behavior is not new, and comparisons between different monitoring systems have already been provided (Bussmann et al., 2001; Mathie, Coster, Lovell, \& Celler, 2004). To measure (limitations of) upper limb activity, several monitoring systems have been used both in populations with upper limb disorders and in groups of healthy subjects, from the perspectives of a variety of research fields (Bernmark \& Wiktorin, 2002; Estill, MacDonald, Wenzl, \& Petersen, 2000; Keil, Elbert, \& Taub, 1999; Patterson et al., 1993; Renfrew et al., 1984; Renfrew, Pettigrew, \& Rapoport, 1987; Taub, Uswatte, \& Pidikiti, 1999; Uswatte et al., 2000; Uswatte et al., 2005; van Hilten et al., 1994; van Vugt, van Hilten, \& Roos, 1996; Vega-Gonzalez \& Granat, 2005). In our opinion, the main advantage of ULAM over these other systems is that it measures a combination of mobility-related activities and upper limb activity, plus the fact that both upper limbs are measured.

A methodological issue that also applies to measurements with ULAM is intrasubject variability of everyday activity, which refers to biological or natural differences in activity patterns of any given person between workdays or weekend days. Between-day variability of upper limb activity may depend on the type of population that is studied, may depend on whether a disorder is acute or chronic, and may differ depending on the outcome measures that are used. From the viewpoint of novelty and patient burden (i.e., not bathing or showering), we chose to perform only 24-h weekday measurements with ULAM for the first application studies. In the near future, we will perform 48-h measurements in order to have some indication of between-day variability of upper limb activity. Also, obtaining norm values for healthy upper limb activity and studying between-day variability of upper limb activity in healthy subjects are very important for future clinical studies with ULAM. When performing a cross-sectional (comparison) study or longitudinal study with ULAM, external factors possibly influencing activity patterns and upper limb activity, such as time of the year/season or family/living situation, should always be considered, because these factors may have an impact on the validity of the results. These factors are similar to the factor of unrepresentativeness of the measurement period (Schasfoort et al., 2006), and all of these factors should be taken into account during time management and planning of studies, selection of control groups, and data analysis.

\section{Current and Future Research Projects}

Development of instruments such as ULAM is an ongoing process of extending possibilities and optimizing current properties. So far, ULAM has been validated and used in descriptive and explorative studies in upper limb CRPS. Currently, a randomized controlled trial is ongoing about the effects of treatment with nitric oxide in upper limb CRPS 1. ULAM is one of the main instruments for objectively assessing changes in arm-hand usage during daily life.

The "classic" AM was not capable of detecting wheelchair driving. In a validity study (Postma et al., 2005), the 
combination of accelerometers at the thighs, trunk, and lower arms appeared to be valid for detecting different types of wheelchair driving, although the sensitive axis of the lower arm sensors had to be changed to the transversal direction. At present, this ULAM configuration is being used in a prospective cohort study of spinal cord injury patients in order to explore the recovery of physical activity during and after the rehabilitation period.

ULAM is also the basis for the development of two more specific upper limb monitors for stroke patients and subjects having repetitive strain injury (RSI). RSI is an umbrella term for multifactorial complaints and disorders of the arm, shoulder, and neck. Variability in body postures and motions (i.e., mobility-related activities) has been described to be a risk factor in the developing of RSI. Therefore, a specific RSI-ULAM configuration has been defined, consisting of acceleration sensors and EMG electrodes on the lower arms, hands, trunk, and leg. This configuration allows measurement at the work place and allows study of the relationship between RSI and variability in postures and motions. For the stroke patients, the ULAM configuration was extended with two electrogoniometers that continuously measured the elbow angle of both arms. Because the movement problems and patterns of stroke patients are different from those of CRPS patients, it was assumed that the ULAM configuration was not sensitive enough to measure the changes and differences accompanying stroke. The validity and sensitivity of this instrument are currently being studied.

The (UL)AM configurations are used mainly to detect mobility-related activities and upper limb activity, but signals can also be used to provide data on the quality (or patterns) of movement. This has been done for walking, is currently being done for the sit-to-stand transfer, and also will be done for the assessment of balance. The signals derived from the lower arms will also contain information on the way the arm was used. For example, in an explorative study, the feasibility of accelerometry for assessing smoothness of movement was examined, and accelerometry was shown to be able to measure smoothness. Further study needs to be done, however.

\section{Conclusion}

ULAM has been proven to have added value for (clinical) application studies in rehabilitation medicine. Its possibilities are not limited to rehabilitation only, however; it also has the potential for a large number of other disciplines and research fields. It has to be noted that it is not the main message of this article to state that ambulatory accelerometry should be regarded as a new reference method or that measuring what a subject actually does during daily behavior is most important. The ULAM technique is a relevant and valuable addition to other techniques currently used in medical and behavioral research: If unobtrusive, objective, and valid measurement of upper limb activity and mobility-related activities during everyday behavior in a subject's personal environment is re- quired, instruments such as ULAM (or AM) should be considered.

\section{REFERENCES}

Bernmark, E., \& Wiktorin, C. (2002). A triaxial accelerometer for measuring arm movements. Applied Ergonomics, 33, 541-547.

Bussmann, H. B., Reuvekamp, P. J., Veltink, P. H., Martens, W. L., \& STAM, H. J. (1998). Validity and reliability of measurements obtained with an "activity monitor" in people with and without a transtibial amputation. Physical Therapy, 78, 989-998.

Bussmann, J. B. J. (1998). Ambulatory monitoring of mobility-related activities in rehabilitation medicine. Rotterdam: Erasmus University Rotterdam.

Bussmann, J. B. J., Martens, W. L. J., Tulen, J. H. M., Schasfoort, F. C., van den Berg-Emons, H. J. G., \& Stam, H. J. (2001). Measuring daily behavior using ambulatory accelerometry: The Activity Monitor. Behavior Research Methods, Instruments, \& Computers, 33, 349-356.

Bussmann, J. B. [J.], Tulen, J. H., van Herel, E. C., \& Stam, H. J. (1998). Quantification of physical activities by means of ambulatory accelerometry: A validation study. Psychophysiology, 35, 488-496.

Bussmann, J. B. [J.], van de LaAR, Y. M., Neeleman, M. P., \& Stam, H. J. (1998). Ambulatory accelerometry to quantify motor behaviour in patients after failed back surgery: a validation study. Pain, 74, 153161.

Bussmann, J. B. J., Veltink, P. H., Koelma, F., van Lummel, R. C., \& STAM, H. J. (1995). Ambulatory monitoring of mobility-related activities: The initial phase of the development of an Activity Monitor. European Journal of Physical Medicine \& Rehabilitation, 5, 2-7.

Estill, C. F., MacDonald, L. A., Wenzl, T. B., \& Petersen, M. R. (2000). Use of accelerometers as an ergonomic assessment method for arm acceleration: A large-scale field trial. Ergonomics, 43, 14301445.

Jain, A., Martens, W. L. J., Mutz, G., Weiss, R. K., \& Stephan, E. (1996). Towards a comprehensive technology for recording and analysis of multiple physiological parameters within their behavioral and environmental context. In J. Fahrenberg \& M. Myrtek (Eds.), Ambulatory assessment: Computer-assisted psychological and psychophysiological methods in monitoring and field studies (pp. 215-236). Seattle: Hogrefe \& Huber.

Keil, A., Elbert, T., \& TAub, E. (1999). Relation of accelerometer and EMG recordings for the measurement of upper extremity movement. Journal of Psychophysiology, 13, 77-82.

Mathie, M. J., Coster, A. C., Lovell, N. H., \& Celler, B. G. (2004). Accelerometry: Providing an integrated, practical method for longterm, ambulatory monitoring of human movement. Physiological Measurement, 25, R1-20.

Patterson, S. M., Krantz, D. S., Montgomery, L. C., Deuster, P. A., Hedges, S. M., \& Nebel, L. E. (1993). Automated physical activity monitoring: Validation and comparison with physiological and self-report measures. Psychophysiology, 30, 296-305.

Postma, K., van den Berg-Emons, H. J., Bussmann, J. B., Sluis, T. A., Bergen, M. P., \& Stam, H. J. (2005). Validity of the detection of wheelchair propulsion as measured with an activity monitor in patients with spinal cord injury. Spinal Cord, 43, 550-557.

Renfrew, J. W., Moore, A. M., Grady, C., Robertson-Tchabo, E. A., CUTLER, N. R., RAPOPORT, S. I., ET AL. (1984). A method for measuring arm movements in man under ambulatory conditions. Ergonomics, 27, 651-661.

Renfrew, J. W., Pettigrew, K. D., \& Rapoport, S. I. (1987). Motor activity and sleep duration as a function of age in healthy men. Physiology \& Behavior, 41, 627-634.

Schasfoort, F. C., Bussmann, J. B. J., Krijnen, H. J., \& Stam, H. J. (2006). Upper limb activity over time in complex regional pain syndrome type 1 as objectively measured with an upper limb-activity monitor: An explorative multiple case study. European Journal of Pain, 10, 31-39.

Schasfoort, F. C., Bussmann, J. B. J., \& Stam, H. J. (2000). Outcome 
measures for complex regional pain syndrome type I: An overview in the context of the international classification of impairments, disabilities and handicaps. Disability \& Rehabilitation, 22, 387-398.

Schasfoort, F. C., Bussmann, J. B. J., \& Stam, H. J. (2002). Ambulatory measurement of upper limb usage and mobility-related activities during normal daily life with an upper limb-activity monitor: A feasibility study. Medical \& Biological Engineering \& Computing, 40, 173-182.

Schasfoort, F. C., Bussmann, J. B. J., \& Stam, H. J. (2004). Impairments and activity limitations in subjects with chronic upper-limb complex regional pain syndrome type I. Archives of Physical Medicine \& Rehabilitation, 85, 557-566.

Schasfoort, F. C., Bussmann, J. B. J., \& Stam, H. J. (2005). Correlation between a novel upper limb activity monitor and four other instruments to determine functioning in upper limb complex regional pain syndrome type I. Journal of Rehabilitation Medicine, 37, 108-114.

Schasfoort, F. C., Bussmann, J. B. J., Zandbergen, A. M. A. J., \& STAm, H. J. (2003). Impact of upper limb complex regional pain syndrome type I on everyday life measured with a novel upper limbactivity monitor. Pain, 101, 79-88.

Taub, E., Uswatte, G., \& Pidikiti, R. (1999). Constraint-induced movement therapy: A new family of techniques with broad application to physical rehabilitation-a clinical review. Journal of Rehabilitation Research \& Development, 36, 237-251.

Uswatte, G., Foo, W. L., Olmstead, H., Lopez, K., Holand, A., \&
SIMms, L. B. (2005). Ambulatory monitoring of arm movement using accelerometry: An objective measure of upper-extremity rehabilitation in persons with chronic stroke. Archives of Physical Medicine \& Rehabilitation, 86, 1498-1501.

Uswatte, G., Miltner, W. H., Foo, B., Varma, M., Moran, S., $\&$ TAUB, E. (2000). Objective measurement of functional upperextremity movement using accelerometer recordings transformed with a threshold filter. Stroke, 31, 662-667.

van DEN Berg-Emons, H. J. G., Bussmann, J. B. J., Balk, A. H. M. M., \& Stam, H. J. (2000). Validity of ambulatory accelerometry to quantify physical activity in heart failure. Scandinavian Journal of Rehabilitation Medicine, 32, 187-192.

van Hilten, B., Hoff, J. I., Middelkoop, H. A., van der Velde, E. A., Kerkhof, G. A., Wauquier, A., ET AL. (1994). Sleep disruption in Parkinson's disease: Assessment by continuous activity monitoring. Archives of Neurology, 51, 922-928.

van Vugt, J. P., van Hilten, B. J., \& Roos, R. A. (1996). Hypokinesia in Huntington's disease. Movement Disorder, 11, 384-388.

Vega-Gonzalez, A., \& Granat, M. H. (2005). Continuous monitoring of upper-limb activity in a free-living environment. Archives of Physical Medicine \& Rehabilitation, 86, 541-548.

(Manuscript received December 16, 2005; revision accepted for publication February 28, 2006.) 